\title{
Tunnel Magnetoresistive Effect in Nanostructured Composite Systems
}

\author{
Stognei O.V.*, Sitnikov A.V., Kalinin Yu.E. \\ Voronezh State Technical University, Voronezh, Russia
}

\begin{abstract}
The results of the investigation of tunnel magnetoresistive effect in a large number of metaldielectric nanocomposites obtained by the same technology and under identical conditions are presented. The composites with multi-elemental amorphous metal phase were considered. It has been shown that the large values of the tunnel magnetoresistive effect in these composites are ensured when the value of the density of electronic states on the Fermi surface is large while other conditions are being equal. The conditions for the appearance of an anomalous positive tunneling magnetoresistance are formulated based on the study of composites with a mono-elemental ferromagnetic phase from cobalt.
\end{abstract}

\section{Introduction}

Nanogranular composites are artificial materials consisting of two different phases (as a rule - from metal and dielectric materials). In a case of relatively low metal concentration (less than 50 at. \%), the metal phase has the form of nano-sized granules randomly distributed in the dielectric matrix. Such morphology determines unusual physical properties of the composites and one of this unique phenomenon is a tunneling magnetoresistance (TMR) [1].

The TMR is due to tunneling of spin-polarized electrons between ferromagnetic nanogranules through dielectric barriers [1]. Owing to this complicated mechanism the TMR is very sensitive to a large number of different factors [2-4]: granule's size distribution, the distance between granules, presence or absence of clusters coexisting together with separated granules, the magnetic anisotropy of granules, parameters of the tunnel barrier, co-tunneling and interface effects etc. All these features can be observed within one composite system when the ratio between metal and dielectric phases is being changed. Being dependent on mentioned factors the tunneling magnetoresistance shows complicated concentration and temperature dependence as well as sensitivity to external influence (heat or electric field treatment) and preparation conditions [5-7].

On the other hand if one compares the maximum values of the TMR in composite systems with different metal phases $(\mathrm{Ni}, \mathrm{Fe}$ or $\mathrm{Co})$ it is possible to see that the TMR differs by more than an order of magnitude [8-11]. The maximum TMR values in Ni-based composites always do not exceed 0.1-0.2\% [8,9], while in Co-based composites the maximum TMR values usually reach 7-8 $\%[10,11]$. Why does it happen? According to the base theories, the value of TMR effect depends on the polarised coefficient of electrons of the metal phase [12] and mutual orientation of magnetic moments of neighbour granules [13]. However experimental data give a more complicated picture. Some experimental results concerning the influence of the metal phase electronic properties as well as composite's morphology on the TMR values are presented in the paper.

\section{Methods}

The presented results have been obtained at the investigation of different composites systems prepared by one deposition equipment and under the same deposition conditions (residual pressure, operating gas pressure, sputter power and so on). All composites have been prepared by an ion-beam sputter of mosaic targets made from a metal base and dielectric plates nonsymmetrically located on its surface. Nonsymmetrical targets give the possibility to obtain samples with the different ratio of metal and dielectric phase in one deposition process and under the same conditions. All studied composites were thin films (3-4 $\mathrm{mkm})$ deposited on glassceramic substrates $(\mathrm{NaCl}$ in a case of HRTM investigation). Magnetoresistance of the samples was measured by the two-probe potentiometric method under a sweeping magnetic field. All measurements were carried out at room temperature.

\section{Results}

\subsection{Composites with amorphous metal granules}

The TMR effect is determined by reorientation of granules magnetic moments under applied magnetic field (it depends on the average angle between the granule magnetic moments) [13]. As a rule, the granules in 
metal-dielectric composites are crystalline and therefore can have different crystalline anisotropy. One can suppose that the anisotropy determines the remagnetization process and influences on the TMR values (for example anisotropy of $\mathrm{Co}$ is much larger than anisotropy of $\mathrm{Ni}$ ). To check this point the composites with amorphous metal granules were studied.

The composites containing metallic phases which can be easily produced in the amorphous state $\left(\mathrm{Co}_{84} \mathrm{Nb}_{14} \mathrm{Ta}_{2}\right.$, $\mathrm{Co}_{41} \mathrm{Fe}_{39} \mathrm{~B}_{20}$ and $\left.\mathrm{Co}_{45} \mathrm{Fe}_{45} \mathrm{Zr}_{10}\right)$ were used for investigation. According to the TEM data, the composites morphology does not depend on the metal phase and is similar in different systems. See the example of TEM photographs for $\left(\mathrm{Co}_{86} \mathrm{Ta}_{12} \mathrm{Nb}_{2}\right)_{34}\left(\mathrm{SiO}_{\mathrm{n}}\right)_{66}$ and $\left(\mathrm{Co}_{41} \mathrm{Fe}_{39} \mathrm{~B}_{20}\right)_{30}\left(\mathrm{SiO}_{\mathrm{n}}\right)_{70}$ composites having the close concentration of the metal phase (fig. 1).

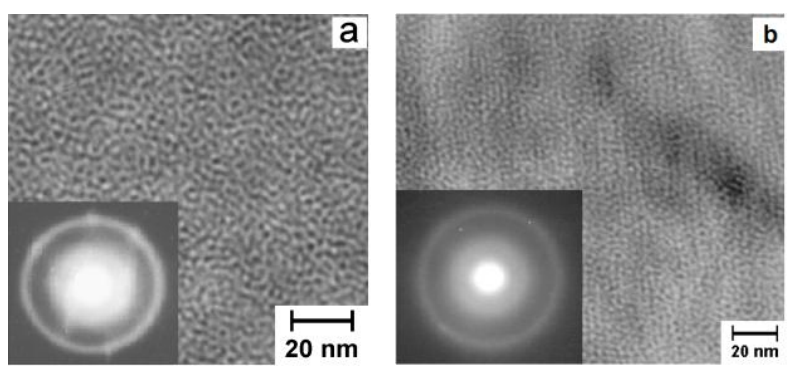

Fig.1. TEM photographs of the composites with different metal phases: a - $\left(\mathrm{Co}_{86} \mathrm{Ta}_{12} \mathrm{Nb}_{2}\right)_{34}\left(\mathrm{SiO}_{\mathrm{n}}\right)_{66} ; \mathrm{b}-\left(\mathrm{Co}_{41} \mathrm{Fe}_{39} \mathrm{~B}_{20}\right)_{30}\left(\mathrm{SiO}_{\mathrm{n}}\right)_{70}$

Besides the absence of the crystalline anisotropy, the metal phases of the studied composites should have similar polarization coefficient of electrons. Really, two metal phases have very close element composition $\left(\mathrm{Co}_{41} \mathrm{Fe}_{39} \mathrm{~B}_{20}\right.$ and $\left.\mathrm{Co}_{45} \mathrm{Fe}_{45} \mathrm{Zr}_{10}\right)$, the third metal phase contains only one ferromagnetic element - cobalt. If we compare the known values of the polarization coefficients for $\mathrm{Co}_{50} \mathrm{Fe}_{50}$ alloy and pure cobalt we will see that the values are close: $50 \%$ for the alloy and $45 \%$ for cobalt [14]. Therefore magnetoresistance of composites with amorphous metal phase should be equal. However, the experiment gives a completely different result (see fig. 2).

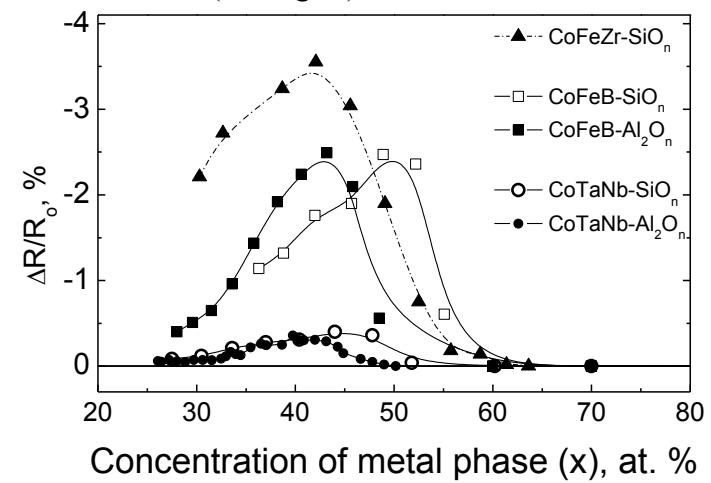

Fig.2. Concentration dependence of the magnetoresistive effect in amorphous granular composites with various combination of metallic $\left[\left(\mathrm{CO}_{86} \mathrm{Ta}_{12} \mathrm{Nb}_{2}\right),\left(\mathrm{CO}_{41} \mathrm{Fe}_{39} \mathrm{~B}_{20}\right)\right.$ and $\left.\left(\mathrm{CO}_{45} \mathrm{Fe}_{45} \mathrm{Zr}_{10}\right)\right]$ and dielectric $\left[\mathrm{SiO}_{\mathrm{n}}\right.$ and $\left.\mathrm{Al}_{2} \mathrm{O}_{\mathrm{n}}\right]$ phases. The magnetoresistance values were determined in a magnetic field of $10 \mathrm{kOe}$.
One should note that all compared composites were obtained by the same equipment and under the same conditions. Maximum TMR values were observed in composites with $\mathrm{Co}_{45} \mathrm{Fe}_{45} \mathrm{Zr}_{10}$ metal phase and minimum values are shown by composites with $\mathrm{Co}_{86} \mathrm{Ta}_{12} \mathrm{Nb}_{2}$ in spite of the fact that composites with metal phase from pure cobalt (the Co-Al-O composites) have high values of the TMR effect (7-8\%) [10,11]. Another important fact is that the TMR values do not depend on the dielectric matrix: composites with different matrix (silicon or alumina oxide) but the same metal phase have equal magnetoresistance (see fig. 2).

Besides the observed difference in TMR values the very unusual correlation was also found in the amorphous nanocomposites: the maximum TMR values, observed in the composites with different metal phase, correlate with the magnitude of saturation magnetostriction of this metal phase (see fig. 3). The magnetostriction in all alloys is positive and in the simplest approach one can suppose that granules are stretched along the applied magnetic field and this makes the distance between granules smaller. The probability of electrons tunnel throw the dielectric barrier exponentially depends on the barrier thickness [1], therefore larger value of magnetostriction leads to the smaller distance between neighbour granules and more intensive electron tunneling when a magnetic field is applied to composite. However, the calculations of the changes in the distance between neighbour granules due to magnetostriction have shown that this effect is negligible (less than 10-13 m) and cannot influence the electron tunneling. Direct estimates of the change of the composite's electrical resistivity based on the tunnel model [1] and taking into account the change in the intergranular distance caused by magnetostriction, also give a negligible value by several orders of magnitude less than the actual TMR values. It is obvious that the mechanism of the observed correlation should have a more fundamental reason.

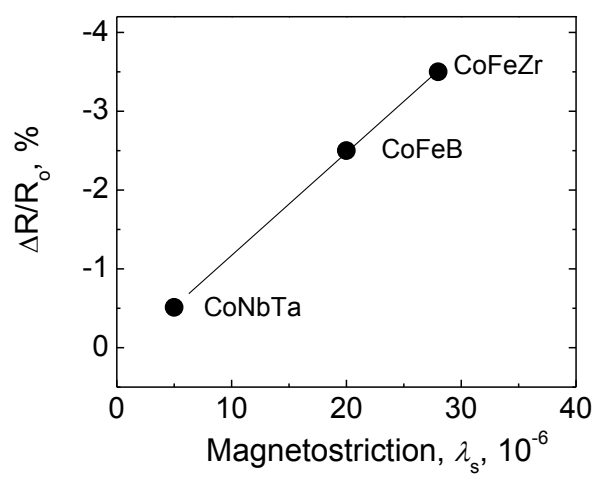

Fig.3. Maximum value of the magnetoresistive effect vs. values of saturation magnetostriction of the metal phase.

Properties of condensed matters associated with charge transport and electronic interaction are determined by the electrons which have energy close to the Fermi level [15] (even the value of magnetostriction effect depends on the density of electronic states [16]). Therefore, the density of electronic states at the Fermi 
level $\left(\mathrm{g}\left(\mathrm{E}_{\mathrm{F}}\right)\right)$ was estimated for the studied composites to explain the obtained result.

The determination was carried out using the " Mott's model » for conductivity in non-metallic systems [15]. The calculation was based on the temperature dependence of composites resistance in the lowtemperature region. In a case of realization of hopping conductivity in the composites one can apply the «Mott's model» and based on the recalculating of the experimental dependencies get the $g\left(E_{F}\right)$ values [17]. The obtained results are presented at the fig. 4 .

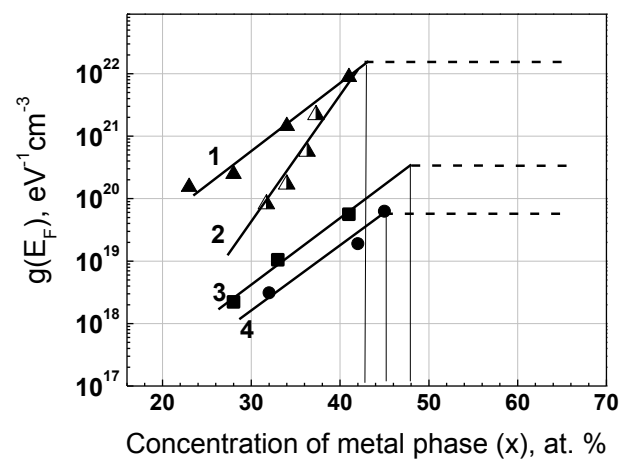

Fig.4. Concentration dependences of the density of electronic states at the Fermi level for different nanocomposites: $1-\left(\mathrm{Co}_{45} \mathrm{Fe}_{45} \mathrm{Zr}_{10}\right)_{\mathrm{x}}\left(\mathrm{SiO}_{\mathrm{n}}\right)_{100-\mathrm{x}} ; 2-\left(\mathrm{Co}_{45} \mathrm{Fe}_{45} \mathrm{Zr}_{10}\right)_{\mathrm{x}}\left(\mathrm{Al}_{2} \mathrm{O}_{\mathrm{n}}\right)_{100-\mathrm{x}}$; $3-\left(\mathrm{Co}_{41} \mathrm{Fe}_{39} \mathrm{~B}_{20}\right)_{\mathrm{x}}\left(\mathrm{SiO}_{\mathrm{n}}\right)_{100-\mathrm{x}} ; 4-\left(\mathrm{Co}_{84} \mathrm{Nb}_{14} \mathrm{Ta}_{2}\right)_{\mathrm{x}}\left(\mathrm{SiO}_{\mathrm{n}}\right)_{100-\mathrm{x}}$. Vertical lines show the concentration of the percolation threshold for each composite

The calculated values indicate the $g\left(\mathrm{E}_{\mathrm{F}}\right)$ in the dielectric matrix of the composites, that is why the $\mathrm{g}\left(\mathrm{E}_{\mathrm{F}}\right)$ values for $\left(\mathrm{Co}_{45} \mathrm{Fe}_{45} \mathrm{Zr}_{10}\right)_{\mathrm{x}}\left(\mathrm{SiO}_{\mathrm{n}}\right)_{100-\mathrm{x}}$ and $\left(\mathrm{Co}_{45} \mathrm{Fe}_{45} \mathrm{Zr}_{10}\right)_{\mathrm{x}}\left(\mathrm{Al}_{2} \mathrm{O}_{\mathrm{n}}\right)_{100-\mathrm{x}}$ are different. As the concentration of the metal increases, the values of $\mathrm{g}\left(\mathrm{E}_{\mathrm{F}}\right)$ also increase and reach values corresponding to the pure metallic phase when metal concentration approaches the percolation threshold. The concentration of the percolating threshold is been pointed by vertical lines in the fig. 4. Since we consider the magnetoresistive effect the only electrons which are in metal phase and directly tunnel from one granule to another one has to be taken into account. Therefore only $\mathrm{g}\left(\mathrm{E}_{\mathrm{F}}\right)$ values of metal phase are important and these values have to be compared if one compare TMR of different composites.

It is obvious, that the density of states determined from the experiments directly correlates to the magnetoresistivity values of the composites (compare fig.2 and fig.4). Thus, to obtain large values of the magnetoresistive effect, not only maximum values of the electron polarization coefficient are needed. The large values of the density of electronic states at the Fermi level are also required.

\subsection{Cobalt based composites and positive TMR}

The technique which has been used to produce the composites allows obtaining samples with very small step in composition. This makes it possible to accurately investigate effects that appear in narrow concentration intervals (for example, near the percolation threshold). It is known that in addition to the usual negative magnetoresistive effect the anomalous positive magnetoresistive effect is observed in the composites with mono-element ferromagnetic phase: $\mathrm{Fe}_{-} \mathrm{SiO}_{2}[18$, 19], $\mathrm{Co}-\mathrm{SiO}_{2}$ [20], $\mathrm{Fe}-\mathrm{Al}_{2} \mathrm{O}_{3}$ [21], Co- $\mathrm{Al}_{2} \mathrm{O}_{\mathrm{n}}$ [22] (see fig. 5).

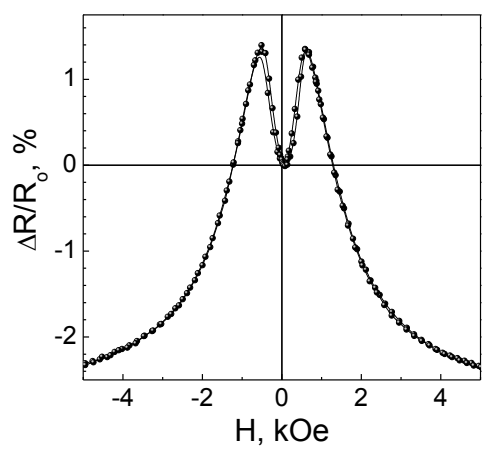

Fig. 5. Example of the positive tunneling magnetoresistance observed in $\mathrm{Co}_{60}\left(\mathrm{Al}_{2} \mathrm{O}_{\mathrm{n}}\right)_{40}$ nanocomposite.

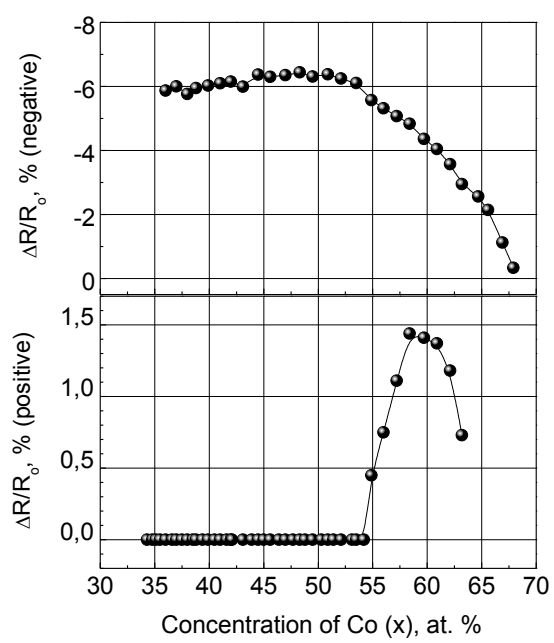

Fig. 6. Concentration dependence of the tunneling magnetoresistance (positive and negative) in $\mathrm{Co}_{\mathrm{x}}\left(\mathrm{Al}_{2} \mathrm{O}_{\mathrm{n}}\right)_{100-\mathrm{x}}$ nanocomposites.

The positive effect is observed only in the vicinity of the percolation threshold (see fig. 6) and has the tunnel origin like the usual negative TMR $[20,22]$. The tunnel nature of the positive effect is confirmed by the nanogranular structure and tunneling conductivity mechanism observed in these composites [20]. Moreover, the positive effect is isotropic: its value and sign do not depend on the mutual orientation of electric current and magnetic field [22], as it could be if the origin of the effect would be different. For example, in a case of anisotropic magnetoresistance, where the mechanism is not a tunnel one, the positive effect is observed only when the current is parallel to the field and the effect is negative when the current is perpendicular to the field $[23,24]$.

The positive isotropic effect is due to the complicated morphology of the composites: simultaneous co- 
existence of relatively large clusters and individual nanogranules $[20,22]$. From one hand the clusters and granules have very different anisotropy and remagnetize under different magnetic fields (clusters need much higher field due to specific chain-like shape [20, 22]). On the other hand, there is a dipole-dipole interaction between clusters and neighbour granules, therefore the field strength where granules are depends on the granules position (how close they are to the cluster). Individual granules remagnetized at the relatively low applied field, but granules need higher field if they are close to clusters and interact with it. As a result, the maximum disorder of the granules magnetic moments (condition for the realisation of maximum resistivity of the composite) takes place at a non-zero magnetic field but at some non zero value. Such situation can take place only when the metal concentration is large i.e. at the threshold.

The question is why the positive magnetoresistance is not observed in every crystalline composite. For example, in Co-CaFn composites, obtained under the same conditions as all other systems, the positive magnetoresistive effect is not observed [25]. Keeping in mind that all composites showing the positive effect have an oxide matrix it could be assumed that positive magnetoresistance is associated with oxygen and partial oxidation of the ferromagnetic granules. Oxidation occurs unevenly and this can lead to the formation of a complicated morphology in the composites. In the absence of an oxide shell the granules can join and form clusters during the growth process, in the presence of an oxide shell on the granules the clusters will not be formed at a given metal concentration. However, studies of the Co-MgFn composite have shown that, despite the absence of the oxygen, the positive magnetoresistance is observed in the samples at the percolation threshold. It is characteristic that in the oxygen-free composites the percolation threshold is shifted toward compositions with the larger concentration of a dielectric phase. This is due to the fact that atoms of the metal phase do not interact with the dielectric and, accordingly, a dielectric layer (the shell) is not formed on the surface of the granules giving the possibility of percolation cluster formation.

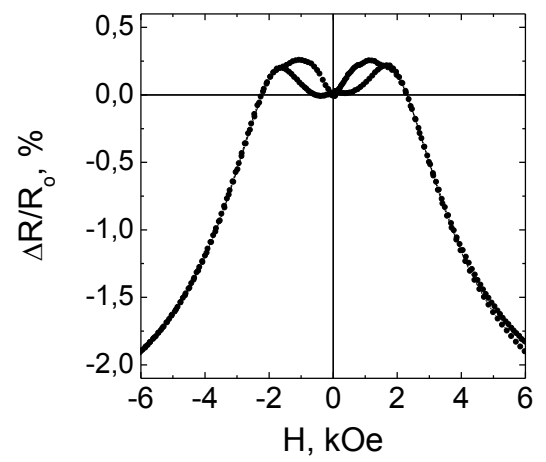

Fig. 7. Example of the positive tunneling magnetoresistance observed in $\mathrm{Co}_{30}\left(\mathrm{MgF}_{\mathrm{n}}\right)_{70}$ nanocomposite

We believe that one of the factors affecting the morphology of composites is the surface energies of the phases forming the composite. The ratio of the surface energies of dielectric $\left(\gamma_{\mathrm{d}}\right)$ and metallic $\left(\gamma_{\mathrm{m}}\right)$ phases affects the granules size, their mutual arrangement and the possibility of clusters formation from several neighbouring granules. This is analogous to the difference in the mechanisms of thin film formation (Frank - Van der Merve or Vollmer - Weber growth mode [26]), depending on the ratio of the surface energies of the substrate and the film. It should be noted that values of the surface energy for metals are always greater than for dielectrics, therefore the dielectric does not form granules in composites with a large content of the metal phase as it occurs with the metal phase at low metal concentration. The difference between the surface energy values of metal and dielectric can vary widely and this affects the morphology of the composite. In the case when the difference $\gamma_{\mathrm{m}}-\gamma_{\mathrm{d}}$ is small, it is advantageous to form a morphology where the growing granules can be joined into clusters having the form of chains from the contacting granules. This mechanism of composite formatting is similar to the Vollmer - Weber mechanism. If the difference $\gamma_{\mathrm{m}}-\gamma_{\mathrm{d}}$ is large, that is the surface energy of the dielectric phase is small, then it is more advantageous to form mutually-isolated granules and this case is similar to the Frank - Van der Merve mechanism for thin films. The study of the structure of $\mathrm{Co}_{55}\left(\mathrm{Al}_{2} \mathrm{O}_{\mathrm{n}}\right)_{45}$ and $\mathrm{Co}_{49}\left(\mathrm{CaF}_{\mathrm{n}}\right)_{51}$ composites is the experimental proof of this assumption (see fig.8).

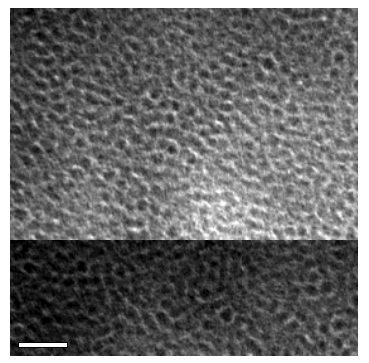

a

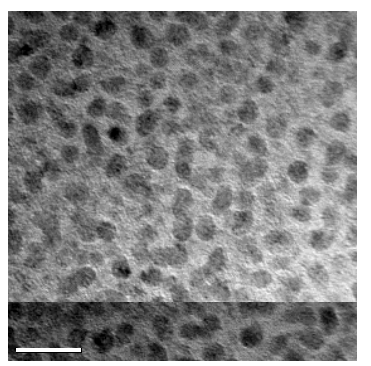

b
Fig.8. TEM photographs of composites with different dielectric phases: a - $\mathrm{Co}_{55}\left(\mathrm{Al}_{2} \mathrm{O}_{\mathrm{n}}\right)_{45} ; \mathrm{b}-\mathrm{Co}_{49}\left(\mathrm{CaF}_{\mathrm{n}}\right)_{51}$. The white tab corresponds to $10 \mu \mathrm{m}$.

The metal phase of both composites is the same and is characterized by a large surface energy $\left(\gamma_{\mathrm{C}_{0}}=2,7 \mathrm{~J} / \mathrm{m}^{2}\right)$ [27], while the values of $\gamma_{\mathrm{d}}$ of the dielectric phases differ significantly: $\left(\gamma_{\mathrm{CaF}}=0,45 \mathrm{~J} / \mathrm{m}^{2}\right.$ [27], $\gamma_{\mathrm{AlO}}=1,4 \mathrm{~J} / \mathrm{m}^{2}$ [27]. It is obvious that the morphology of these two composites is significantly different despite the close composition (the cobalt concentration is similar in the composites). A complex labyrinth-like structure with clusters in the form of chains of contacting granules is observed in the $\mathrm{Co}_{55}\left(\mathrm{Al}_{2} \mathrm{O}_{\mathrm{n}}\right)_{45}$ composite. A similar structure was observed in composites with $\mathrm{SiO}_{2}$ matrix [20]. In the $\mathrm{Co}_{49}\left(\mathrm{CaF}_{\mathrm{n}}\right)_{51}$ composite the morphology is different: the granules are larger in size and, most importantly, they are isolated from each other and do not form any clusters. We believe that the observed difference in morphology is a consequence of the low value of the surface energy of magnesium fluoride. Such an unrelated arrangement of the granules does not create the 
conditions for the appearance of the positive magnetoresistance. It should be noted that there is no positive magnetoresistance in the $\mathrm{Co}_{49}\left(\mathrm{CaF}_{\mathrm{n}}\right)_{51}$ composites.

\section{Conclusion}

The value of tunnel magnetoresistive effect in metaldielectric nanocomposites is determined not only by the polarizing coefficient but also by the density of electronic states on the Fermi level as well.

Difference between the surface energy of metallic and dielectric phases determines the nanocomposites morphology. The large difference (in a case of small surface energy of the dielectric phase) provides the mutual separation of metal granules even at big metal concentration.

\section{References}

1. B.Abeles, P. Sheng M.D.Coutts and Y. Arie Advances in Physics 24, 407 (1975)

2. V.I.Kozub, A.V.Shumilin Solid State Communications 171, .55 (2013)

3. A.Ya.Vovk, V.O.Golub et al. JMMM 272-276, E1403 (2004)

4. L.Kraus, O. Chayka et al. JMMM 226-230, 669 (2001)

5. T.N. Koltunowicz, P. Zukowski et al. Vacuum 120 44 (2015)

6. A.Svito, A. K.Fedotov et al. J. Alloys and Comp. 699, 818 (2017)

7. O.V. Stognei, V.A. Slyusarev et al. Microelectronics Engineering 69, 476 (2003)

8. A.Gerber, A.Milner, B.Groisman et al. PR B 55, 6446 (1997)

9. O.V.Stognei, A.J. Al-Maliki et al. Semiconductors 50, 709 (2016)

10. S.Sankar, D.Dender, J.A.Borchers et al. JMMM 221, 1 (2000)

11. S.Mitani, H.Fujimori, S.Ohnuma JMMM 165, 141 (1997)

12. M.Julliere Phys.Let. 54A, 225 (1975)

13. J.C.Slonczewski PR B, 39, 6995 (1989)

14. J.S.Moodera, G.Mathon JMMM 200, 248 (1999)

15. N. F. Mott, E.A. Davis, Electronic Processes in Non-Crystalline Materials (Clarendon Press Oxford, 1971)

16. I.Yoshikazu, N. Miura Physics and Engineering Applications of Magnetism (Springer, 1991)

17. Y.E.Kalinin, A.N.Remizov, A.V.Sitnikov, Physics of the Solid State 46. 2176 (2004)

18. A. E. Varfolomeev, M. V. Sedova Physics of the Solid State 45, 529 (2003)
19. B. A. Aronzon, D. Yu. Kovalev, A. E. Varfolomeev Physics of the Solid State 41, 857 (1999)

20. S. Sankar, A.E. Berkowitz, D.J. Smith. Phys.Rev. B62, 14273 (2000)

21. M.A.S. Boff, J. Geshev, J.E. Scmidt. J. Appl.Phys. 91, 12, 9909 (2002)

22. O. V. Stognei, A. V. Sitnikov, Yu. E. Kalinin, S. F. Avdeev, M. N. Kopytin Physics of the Solid State 49, 164 (2007)

23. K. Y. Wang, K. W. Edmonds et al. PR B 72, 085201 (2005)

24. A. Grebennikov, O. Stognei Solid State Phenomena 233-234, 501 (2015)

25. O.V. Stognei, A.V. Sitnikov, S.F. Avdeev, Yu.E Kalinin CNM Int. Conf. 114 (2006)

26. Pimpinelli, Alberto; Jacques Villain. Physics of Crystal Growth. (Cambridge: Cambridge University Press, 1998)

27. F.J.Himpsel, J.E.Ortega et. al. Advances in Physics 47, 511 (1998) 